\title{
ELM mitigation by nitrogen seeding in the JET gas box divertor
}

\author{
J Rapp ${ }^{1}$, T Eich ${ }^{1}$, M von Hellermann ${ }^{2}$, A Herrmann ${ }^{3}$, L C Ingesson ${ }^{2}$, \\ S Jachmich ${ }^{4}$, G F Matthews ${ }^{5}$, V Philipps ${ }^{1}$, G Saibene ${ }^{6}$ and contributors \\ to the EFDA-JET Workprogramme \\ ${ }^{1}$ Institut für Plasmaphysik, Forschungszentrum Jülich GmbH, EURATOM Association, TEC, \\ Jülich, Germany \\ ${ }^{2}$ FOM Instituut for Plasmafysica Rijnhuizen, Association EURATOM-FOM, TEC, Nieuwegein, \\ The Netherlands \\ ${ }^{3}$ Max-Planck Institut für Plasmaphysik, EURATOM Association, Garching, Germany \\ ${ }^{4}$ LPP-ERM/KMS, EURATOM-Belgian State Association, TEC, Brussels, Belgium \\ 5 EURATOM-UKAEA/Fusion Association, Culham Science Center, Abingdon, OX14 3DB, UK \\ ${ }^{6}$ EFDA, CSU-Garching, Garching, Germany
}

Received 25 October 2001, in final form 24 January 2002

Published 22 May 2002

Online at stacks.iop.org/PPCF/44/639

\begin{abstract}
One of the most severe problems for fusion reactors is the power load on the divertor target plates. Technically only power loads of less than $10 \mathrm{MW} \mathrm{m}^{-2}$ are acceptable. However, strong edge localized mode (ELM) activity can lead to power loads in excess of $800 \mathrm{MW} \mathrm{m}^{-2}$. In order to reduce the steady-state heat flux and the transient heat flux due to ELMs, radiation cooling experiments were performed at JET. Nitrogen was puffed into the divertor up to a radiative power fraction of $90 \%$. This was achieved at a density of 0.85 times the Greenwald density (Greenwald M 1988 Nucl. Fusion 28 2199), while maintaining an $\mathrm{H}$-factor of $f_{\mathrm{H} 98}=0.82$. The $Z_{\text {eff }}$ in all those discharges stayed around 2.0. At approximately $55 \%$ radiative power fraction, the ELM characteristic changes from type I to type III, resulting in a loss of confinement of about $25 \%$ due to a degradation of the edge pedestal and hence a reduction of the ELM power load to the divertor tiles. By increasing the radiative power fraction to values of about $90 \%$, the heat flux is reduced to $2 \mathrm{MW} \mathrm{m}^{-2}$.
\end{abstract}

\section{Introduction}

Radiation cooling experiments with extrinsic impurities have been performed on several tokamaks [2-4] in order to reduce the heat flux to the plasma facing components such as limiters or divertors. For ITER, average steady-state heat fluxes below $10 \mathrm{MW} \mathrm{m}^{-2}$ have to be obtained, which requires a very shallow angle of incidence and cooling due to radiation.

7 See the appendix of the paper by J Pamela, Overview of recent JET results Proc. IAEA Conf. on Fusion Energy (Sorrento, 2000). 
Due to the strong transient heat loads, type-I ELMy H-modes are eventually unacceptable as an operating scenario for ITER. The transient heat load on the divertor has to be lower than $\approx 40 \mathrm{MJ} \mathrm{m}^{-2} \mathrm{~s}^{-1 / 2}$ for carbon tiles (ablation) and lower than $\approx 65 \mathrm{MJ} \mathrm{m}^{-2} \mathrm{~s}^{-1 / 2}$ for tungsten tiles (melting), otherwise the lifetime of the divertor will be reduced to a few hundred pulses. The solution to the problem of transient heat loads might be the operation in H-modes with type-II ELMs or small type-III ELMs. Since type-II ELMs have only been found at rather large, ITER irrelevant $q_{95}$ of $\geqslant 3.8$ and close to double null divertor operation [5] (which is also not considered to be an operating scenario for the present ITER design), emphasis is here taken to the type-III ELMy H-mode.

In addition to the reduction of the transient heat loads, a reduction of the temperature in front of the divertor target below $10 \mathrm{eV}$ is necessary in order to be compatible with the application of a tungsten divertor. Physical sputtering might result in concentrations of tungsten in the plasma core leading to non-acceptable radiated power fractions from the plasma core. For ITER, a tolerable tungsten concentration of $2 \times 10^{-5}$ is predicted [6]. For these reasons it has to be demonstrated that an operation with a partial detached divertor and target temperatures of several $\mathrm{eV}$ is feasible, since the erosion yields due to the impact of light impurities with a tungsten target drop drastically below $10 \mathrm{eV}$ [7].

Extensive studies on nitrogen seeded discharges in the JET Mk-I, Mk-IIA and Mk-IIAP divertors have been reported by Matthews et al [4]. In this paper, the application of nitrogen seeding within a type-III ELMy H-mode scenario in the closed JET gas box divertor Mk-IIGB is studied. The JET Mk-IIGB is the most closed one in a series of divertors, which were tested at JET. In figure 1 all JET divertors are displayed. This 'gas box' type geometry and the vertical targets are also foreseen for ITER.

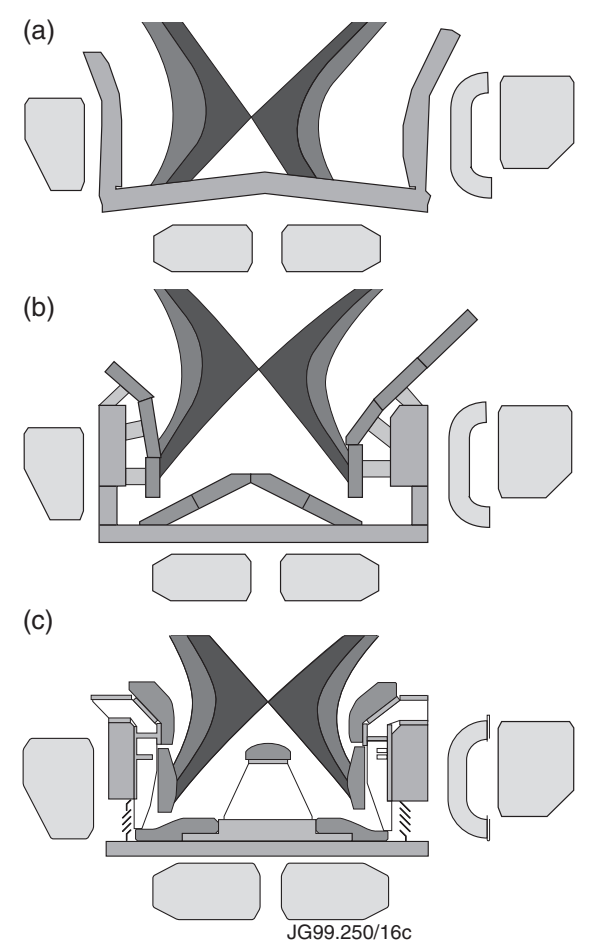

Figure 1. JET divertors: (a) Mk-I; (b) Mk-IIA, Mk-IIAP; (c) Mk-IIGB. 


\section{Radiation cooling by nitrogen seeding in the JET gas box divertor}

At JET, many impurity seeding experiments have been performed $[4,9]$. In this paper, experiments with nitrogen seeding are reported. Nitrogen is a low- $Z$ impurity, which has its maximum in the cooling rate (coronal equilibrium) at $\approx 10 \mathrm{eV}$ [8]. Hence it is radiating at these low temperatures which are typical in the divertor. At temperatures above $2 \mathrm{keV}$ it is totally ionized, thus leading to little radiation from the plasma core. Furthermore, nitrogen is a non-recycling gas, which will minimize migration from the divertor region to the main chamber region. Indeed, it was shown that increasing $Z$ by using neon or argon instead of nitrogen, the ratio of the divertor radiation to the bulk radiation is decreasing [4]. This altogether gives hope that nitrogen is optimal to localize the radiation in the divertor without influencing the plasma core too much.

In figure 2, an overview of a typical discharge (2.5 MA, $2.4 \mathrm{~T}$, high clearance equilibrium, vertical target, $q_{95}=2.8$ ) is shown (\#53318). Deuterium and nitrogen are fuelled with $\Gamma=3 \times 10^{22} \mathrm{el} \mathrm{s}^{-1}$. At a radiative power fraction of 55\%, the ELM characteristic changes from type-I to type-III ELMs. At the same time, the confinement drops from $f_{\mathrm{H} 98}=1.0$ to $f_{\mathrm{H} 98} \approx 0.8$. The density and radiative power fractions are further increased, finally leading to $N^{\mathrm{GW}}=0.85$ and $f_{\text {rad }}=0.9$. During this time the stored energy is increasing, consistent with the density dependence in the ITB98 ELMy H-mode scaling law. $Z_{\text {eff }}$ is increasing for the duration of the seeding up to 2.1. At $19.5 \mathrm{~s}$ the total radiative power reaches its peak value $(90 \%)$. Most of the radiation originates from the X-point region in this detached case as the tomographic reconstructions show (figure 3), whereas in the attached case significant

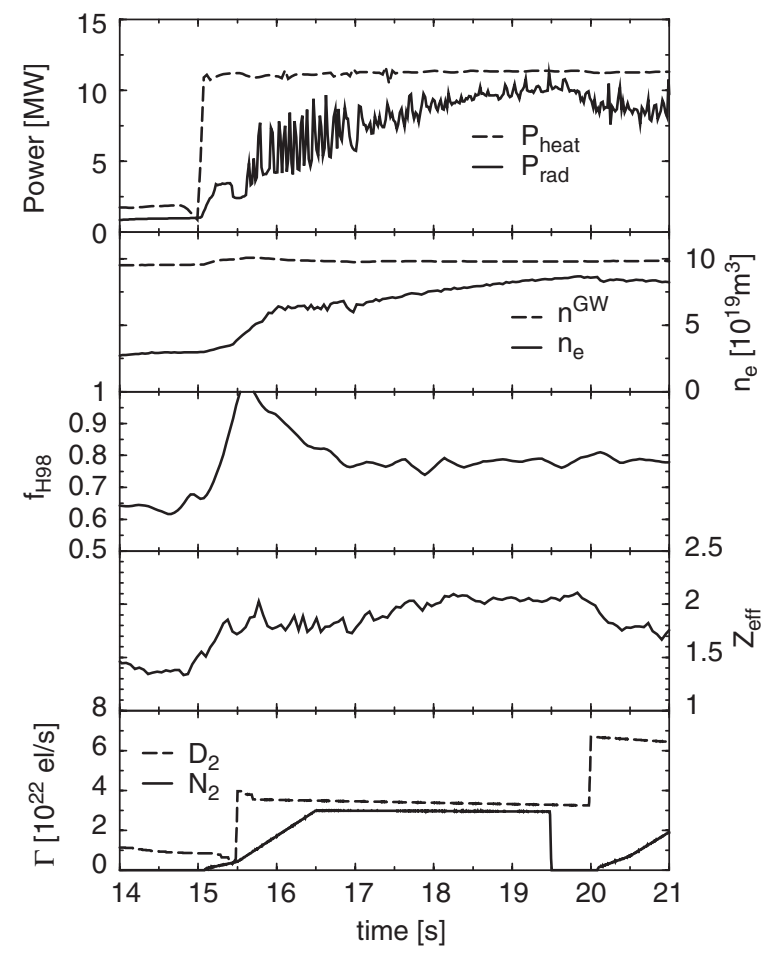

Figure 2. \#53318: overview over discharge; $I_{\mathrm{p}}=2.5 \mathrm{MA}, B_{\mathrm{t}}=2.4 \mathrm{~T}, \delta=0.2$, high clearance equilibrium, vertical target. 

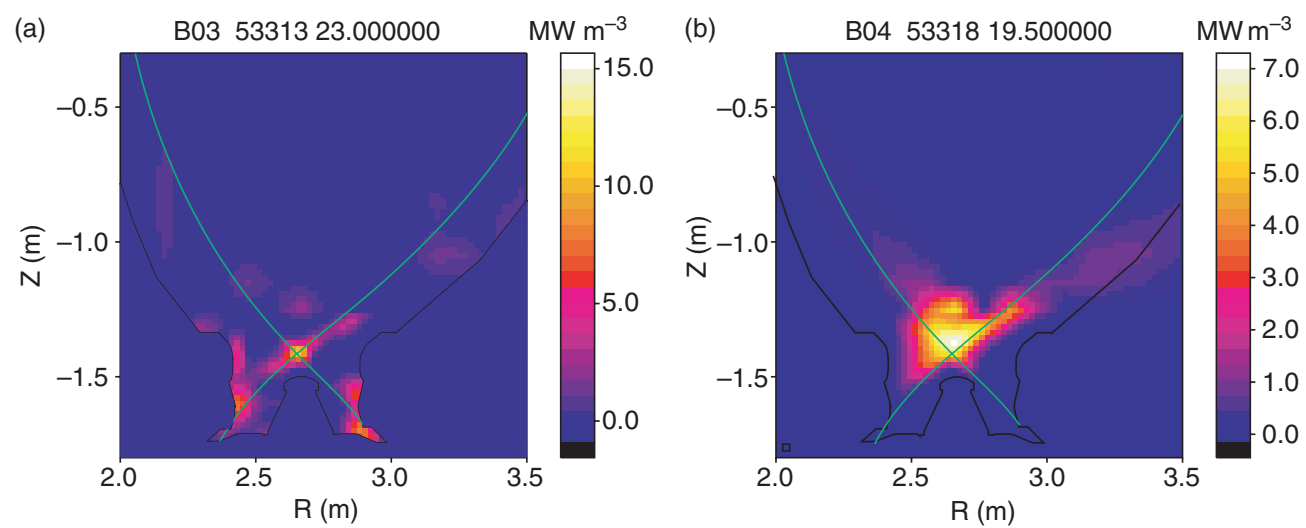

Figure 3. Tomographic reconstructions of the total radiated power derived from bolometry. (a) \#53313, 23 s: attached divertor conditions with type-I ELMs, averaged over type-I ELMs, $40 \%$ radiative power fraction; (b) $\# 53318,19.5 \mathrm{~s}$ : detached divertor conditions with type-III ELMs, averaged over type-III ELMs, $90 \%$ radiative power fraction.

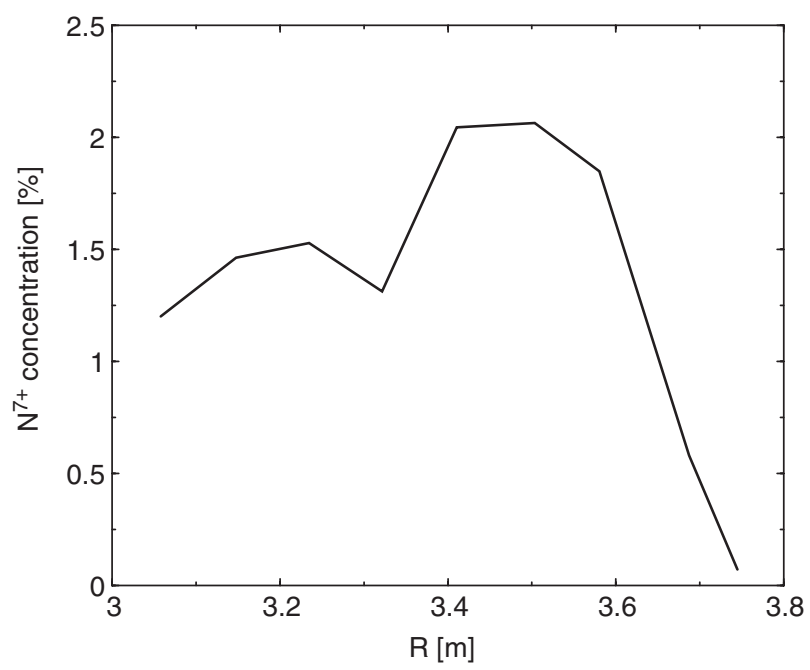

Figure 4. \#53318, $t=19.5 \mathrm{~s}$ : nitrogen concentration profile as derived from CXRS.

radiation is also encountered from the vicinity of the target plates. During the whole discharge, regular sawtooth activity is observed and no accumulation of nitrogen on axis was encountered, as the hollow nitrogen concentration profile at the highest radiation level demonstrates (figure 4).

\subsection{Power balance}

In order to prove the effect of radiation cooling, it has to be shown that the thermal load on the divertor tiles is indeed significantly reduced. For pulse $\# 53318$, in which a maximum radiated power fraction of $90 \%$ was achieved, an energy balance was performed by balancing the input energy with the radiated and convected energy $\left(W_{\text {in }}=W_{\text {rad }}+W_{\text {conv }}\right)$ during the diverted discharge phase. The total radiated energy is determined by the bolometers. The convected 
energy is derived by the divertor thermocouples and taking into account the absorption of radiated energy on the divertor tiles $\left(W_{\text {rad,div }}\right)$. The ratio between the power radiated onto the divertor tiles $\left(P_{\text {rad,div }}\right)$ and the total radiated power $\left(P_{\text {rad }}\right)$ is derived from tomographic reconstructions of the multi-chord bolometer diagnostic. After the local emissivity is obtained, the wall load of each individual wall segment is calculated taking into account the full geometry of the vacuum vessel. In order to minimize the effect of uncertainties in the divertor region, resulting from very local radiation zones, the heat load due to electromagnetic radiation is integrated over all divertor tiles. The JET divertor is equipped with 22 thermocouples from which the total absorbed energy on the divertor tiles for a complete discharge is derived $\left(W_{\text {div }}=W_{\text {conv }}+W_{\text {rad,div }}\right)$. A remarkable agreement is found for the energy $W_{\text {div }}$ derived from thermocouples (67.6 MJ) and derived from the input energy and radiated energy (67.4 MJ), indicating that those high radiative power fractions are reliable.

For a more detailed power balance, IR thermography is necessary. Since the thermography system in the JET gas box divertor has a complicated periscope view and the septum is vignetting large fractions of divertor region, not all configurations are suitable for a reliable measurement. For this reason, a configuration with upward shifted strike zones has been chosen in order to optimize the IR thermography analysis [10]. For such an optimized configuration (\#53772), a power balance has been made. Nitrogen is seeded up to a total radiated power fraction of $80 \%$. The temporal evolution of the heating power $\left(P_{\text {heat }}\right)$, the change in the stored energy $\left(\mathrm{d} W_{\mathrm{dia}} / \mathrm{d} t\right)$, the total radiated power onto the main chamber walls ( $P_{\text {rad,bulk }}=P_{\text {rad }}-P_{\text {rad,div }}$ ) as derived from tomography and the total heat flux on the divertor tiles $\left(P_{\text {div }}\right)$ derived from thermography are shown in figure 5. The model used to calculate the heat flux from the surface temperature measured by IR thermography is described in detail in [11]. The total power on the divertor tiles $\left(P_{\text {div }}\right)$ is calculated taking into account a toroidal wetted fraction of TWF $=90 \%$, which is typical for the used vertical target configuration with an edge safety factor of $q_{95}=2.9$. In general, the power balance $P_{\text {bal }}=P_{\text {heat }}-P_{\text {rad,bulk }}-P_{\text {div }}-\mathrm{d} W_{\text {dia }} / \mathrm{d} t$ is consistent within the experimental error bars $( \pm 1 \mathrm{MW})$. Only in the beginning of the auxiliary heated discharge period at the $\mathrm{L}$ to $\mathrm{H}$ transition and later in the discharge at the transition from type-I to type-III ELMs a deviation of $\approx 2 \mathrm{MW}$ occurs, for which the reason is still unclear.

This satisfactory power balance elucidates the effect of the radiation cooling. The steadystate total power deposited in the divertor is reduced to $50 \%$ at a radiative power fraction of $80 \%$. During the type-III ELM phase, the electron temperature in front of the target is well below $10 \mathrm{eV}$ in between ELMs. As is shown in the following paragraphs, the transient heat load is also reduced significantly. This altogether is a successful demonstration of the radiation cooling.

\subsection{Detachment in between ELMs}

For JET, a general approach for the characterization of the divertor detachment was developed $[12,4]$. This criterion, degree of detachment (DoD), compares the expected ion saturation current $I_{\mathrm{s}}^{\text {scal }}$ for attached conditions, as derived from a simple two-point model, with the actual measured ion saturation current $I_{\mathrm{s}}^{\text {meas }}$. When the deviations between both are larger than certain values, the divertor is detached. The expected ion saturation current scales like $I_{\mathrm{s}}^{\text {scal }} \propto \bar{n}_{\mathrm{e}}^{2} /\left(1-f_{\mathrm{rad}}\right)$, with $f_{\text {rad }}$ being the radiative power fraction. If the $\operatorname{DoD}\left(=I_{\mathrm{s}}^{\mathrm{scal}} / I_{\mathrm{s}}^{\text {meas }}\right)$ is larger than 2 for the peak ion flux or larger than 10 for the integrated ion flux, the divertor is considered to be completely detached [12]. It is important to note that for the high frequent type-III ELMs present here, the time in between ELMs is with $3 \mathrm{~ms}$ (see also figure 8) significantly larger than the particle dwell time in the SOL with 0.4-1 ms. This means that 


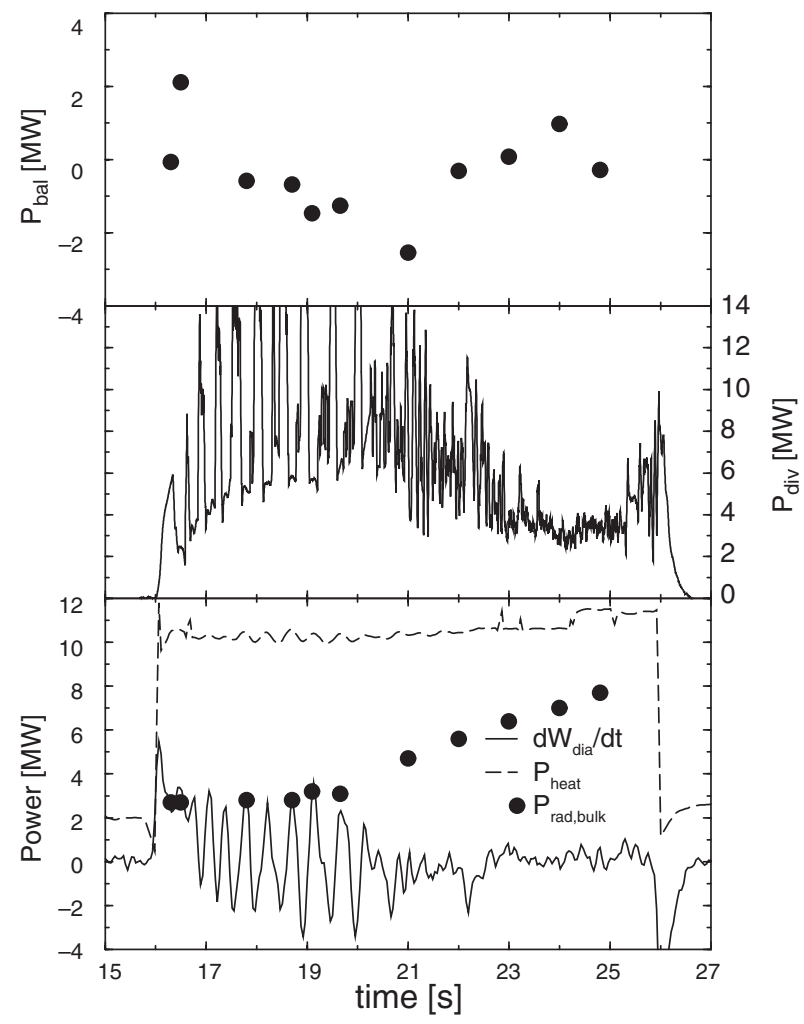

Figure 5. \#53772: power balance $P_{\text {bal }}$ as derived from IR thermography $P_{\text {div }}$, bolometric reconstructions $P_{\text {rad,bulk }}$, heating power $P_{\text {heat }}$ and the change in diamagnetic energy $\mathrm{d} W_{\mathrm{dia}} / \mathrm{d} t$; $P_{\text {bal }}=P_{\text {heat }}-P_{\text {div }}-P_{\text {rad,bulk }}-\mathrm{d} W_{\text {dia }} / \mathrm{d} t$.

the SOL is equilibrated in between ELMs, which is a prerequisite for the application of the two-point model in the conduction-limited regime.

The steady state of the divertor is determined by the particle and heat flux in between ELMs. The DoD, as derived from the lower envelope of the ion saturation current $\left(I_{\mathrm{s}}\right)$, is shown in figure 6. When the H-mode plasma has type-I ELMs, the divertor is attached. After the transition from type-I ELMs to type-III ELMs, the DoD ${ }^{\text {peak }}$ becomes 10 and the integrated DoD ${ }^{\text {int }}$ becomes 20 at the inner divertor. At the strike point of the outer divertor, the DoD as well reaches values $\geqslant 6$. This means that as soon as type-III ELMs are present, at least the inner divertor is completely detached in between ELMs. In figure 7, the DoD for the outer divertor is shown as a function of the confinement enhancement factor, which illustrates that detachment is only achieved in type-III ELMy H-modes leading simultaneously to lower confinement.

\section{ELM mitigation}

In the previous section, it was shown that the total power flux to the divertor target can be reduced significantly. Nevertheless, the essential question with respect to the success of the radiation cooling is related to the ELM power deposition. Is it possible to actually buffer ELMs by the gas target or radiate large fractions of the ELM energy before hitting the target? Furthermore, is it possible to reduce the electron temperature in front of the target plate also transiently during ELMs below $10 \mathrm{eV}$ ? 


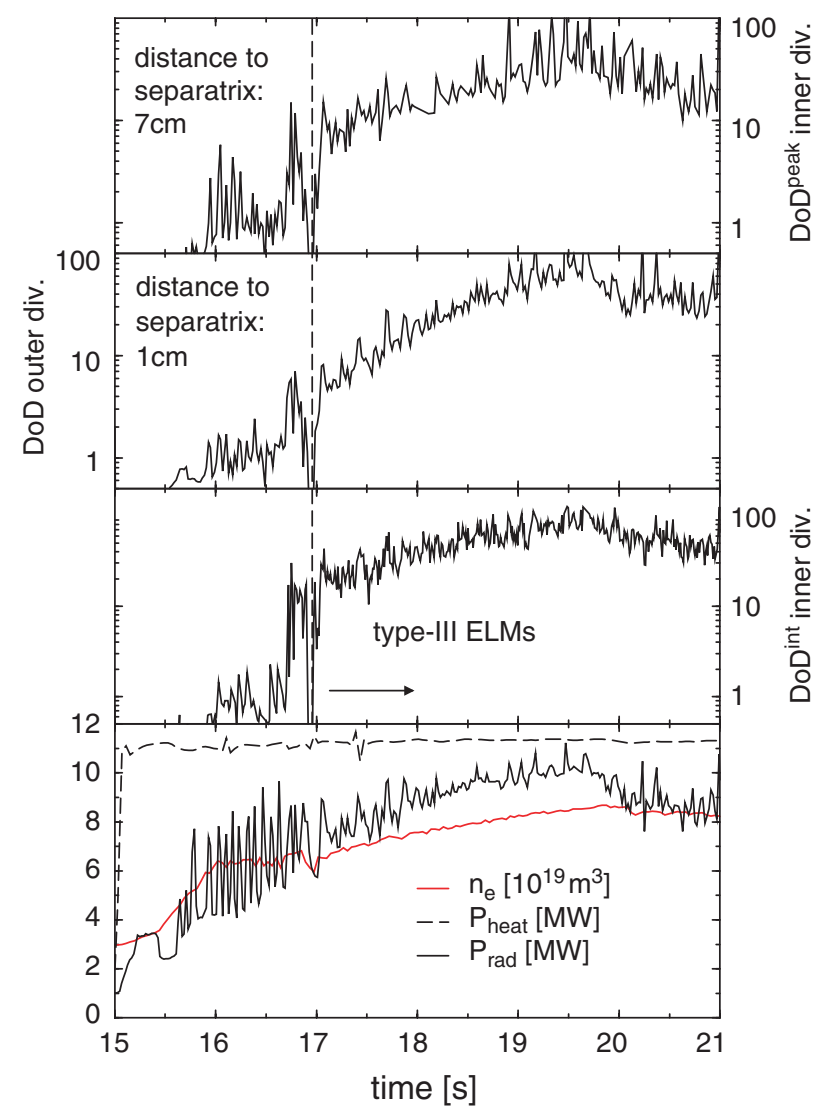

Figure 6. \#53318: DoD at inner and outer divertor target, peak ion flux DoD ${ }^{\text {peak }}$, integrated ion flux DoD ${ }^{\text {int }}$.

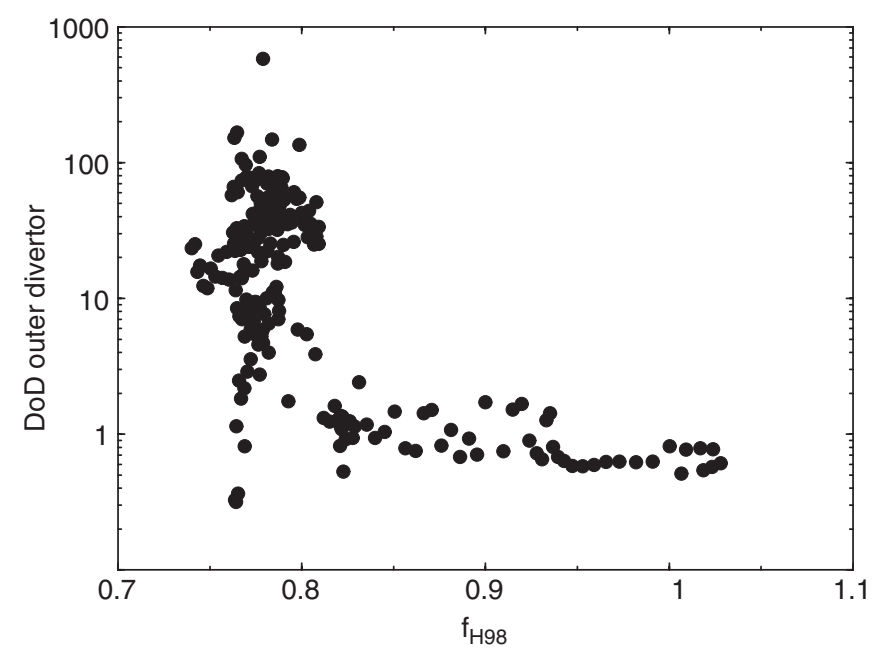

Figure 7. \#53318: DoD at outer divertor target strike zone versus confinement enhancement factor. 
Unfortunately, due to technical reasons (vignetting effects in the Mk-IIGB divertor), good measurements with IR thermography and measurements with the fixed Langmuir probes are mutually exclusive. For this reason, information on ELMs from Langmuir probe measurements is extracted from pulse \#53318 (maximum 90\% radiation), and for IR thermography pulse \#53772 (maximum $80 \%$ radiation) is used.

The picture of the detachment is more complicated during the actual ELM event. Figure 8 shows the upper envelope of the ion saturation current at the outer strike zone $I_{\mathrm{s}}$, meaning the amplitude of the ELMs, and the ELM frequency. By increasing the radiation level, the amplitude of $I_{\mathrm{S}}$ is decreasing while the ELM frequency is increasing up to $300 \mathrm{~Hz}$. The increase of the ELM frequency is usually accompanied by a loss in confinement. More precisely, $f_{\mathrm{ELM}}$ is a consequence of the pedestal pressure and the inter-ELM transport. A simple model, introduced by Fishpool [13], describes the relationship between the energy loss at the pedestal $\Delta W, f_{\mathrm{ELM}}$ and the energy confinement time $\tau_{E}$ :

$$
\frac{\Delta W}{W}=\frac{1}{b} \frac{P}{P_{\text {in }}}\left(1-\mathrm{e}^{-b / f \tau_{E}}\right)
$$

with $P$ the power crossing the pedestal, $P_{\text {in }}$ the total input (heating) power, $b=\tau_{E} / \tau=14$, and $\tau$ being the reheat time in between ELMs. In figure 8 this $\Delta W / W$ scaling is shown with experimental data for $f_{\mathrm{ELM}}$ and the thermal energy confinement time $\tau_{E}$. The power crossing the pedestal $P$ was corrected for radiation from the plasma core during the ramp up of the radiated power fraction. Due to the nitrogen seeding a reduction of the edge pedestal is expected, leading to lower ELM energy losses as seen in figure 8. In the following, this $\Delta W / W$ scaling will be compared to the heat flux density arriving at the target.

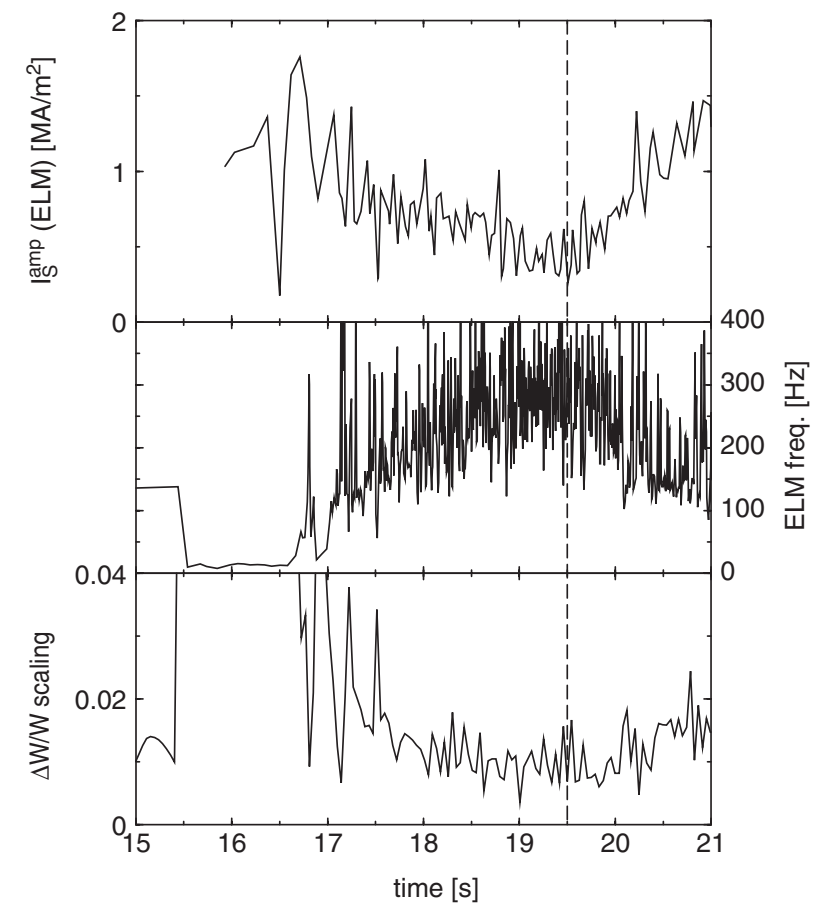

Figure 8. \#53318, outer divertor strike zone: upper envelope of ion saturation current, meaning ELM amplitude; ELM frequency; $\Delta W / W$ scaling, predicted; the vertical dashed line indicates the time of $90 \%$ radiative power fraction. 
Together with the ion saturation current, the electron temperature in front of the target plate is decreasing strongly with increasing radiation level. At the highest level of radiation during the type-III ELMs, the peak electron temperature, measured in front of the target plate, is reduced to $10 \mathrm{eV}$. From the Langmuir probes also the parallel heat flux density can be derived: $q_{\|}=j_{\mathrm{s}}\left(7.8 T_{\mathrm{e}}+E_{\mathrm{rec}}\right)$, with $E_{\mathrm{rec}}=17.8 \mathrm{eV}$ being the ionization energy $(13.6 \mathrm{eV})$ and the recombination energy $(4.2 \mathrm{eV})$ of the deuterons which recombine to molecules at the wall. In order to be comparable to IR measurements, this parallel heat flux is recalculated into a perpendicular heat flux to the target surface flux by taking into account the pitch angle of the field line to the target plate (figure 9). The peak heat flux density (during ELMs, upper envelope) is reduced during the highest radiative power fractions down to $P_{\text {target }} \approx 2 \mathrm{MW} \mathrm{m}^{-2}$. This is in reasonable agreement with IR thermography measurements, which have been taken in a similar pulse (\#53772). Figure 10 shows power flux derived from IR thermography reducing down to $P_{\text {target }} \approx 1.5 \mathrm{MW} \mathrm{m}^{-2}$. Taking into account the ELM duration of $0.4 \mathrm{~ms}$ leads to a power flux of $P_{\text {target }} \approx 5 \pm 2 \mathrm{MW} \mathrm{m}^{-2}$ at a radiative power fraction of $80 \%$. The slightly higher power flux derived from IR thermography as compared to the power flux derived from Langmuir data could be due to a higher relative contribution of the ions to the power flux during ELMs. However, this strong reduction in the power flux to the target (approximately a factor of 3 ) is not consistent with the $\Delta W / W$ scaling. Figure 11 shows the ratio of the power to the outer target to the $\Delta W / W$ scaling as a function of the radiated power fraction. Although there is a large scatter in the data, clearly a trend to higher radiated power fractions can be extracted. The reduction of the power to the target is stronger as the scaling predicts

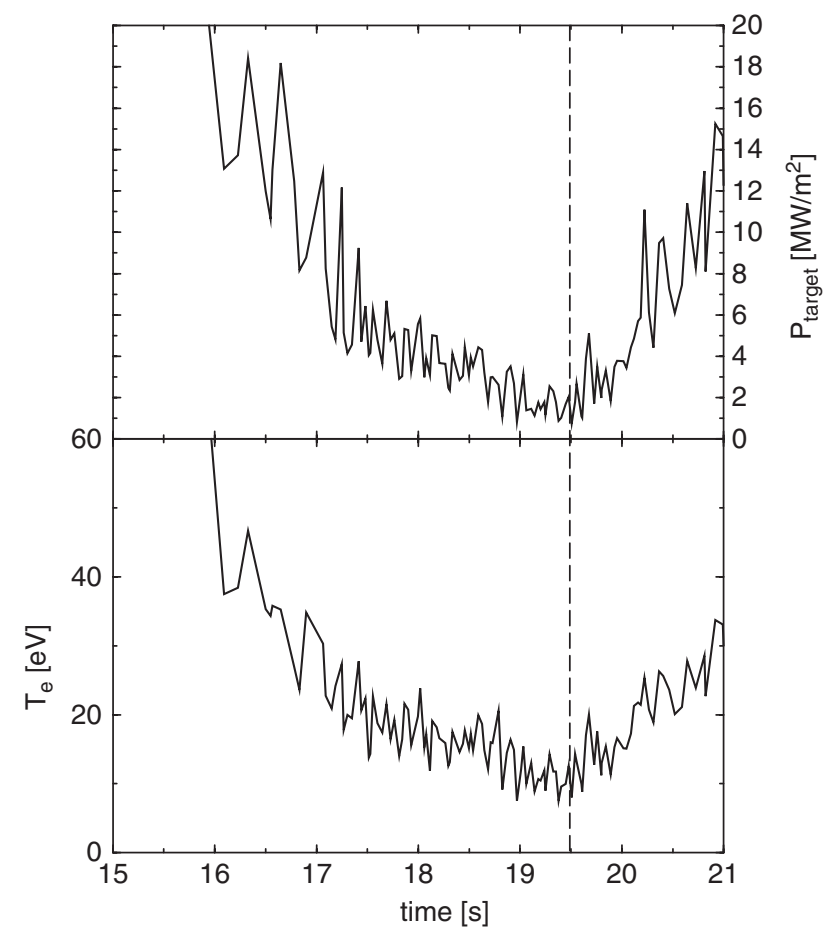

Figure 9. \#53318, outer divertor strike zone: target heat flux density as derived from Langmuir probes; electron temperature in front of the target plate, upper envelope; the vertical dashed line indicates the time of $90 \%$ radiative power fraction. 


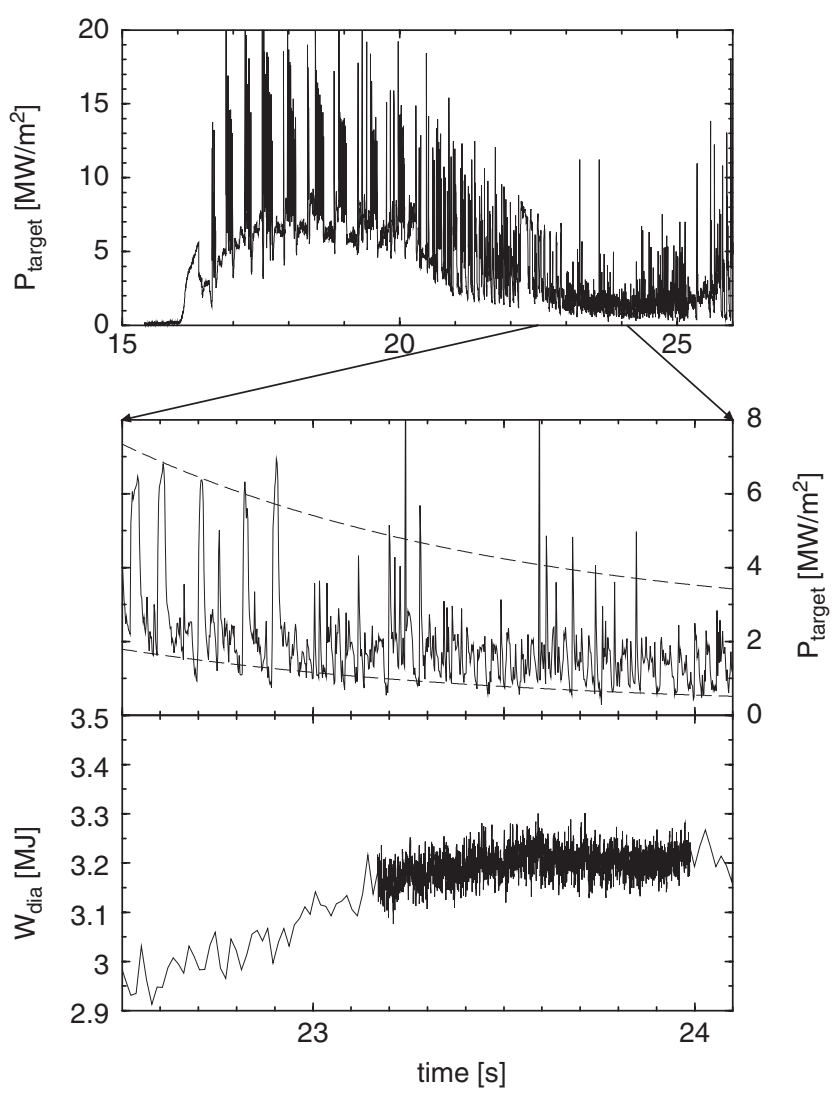

Figure 10. \#53772, outer divertor strike zone: target heat flux density as derived from IR thermography ( $2 \mathrm{~ms}$ integration), the dashed lines should guide the eyes for an approximate upper and lower envelope; diamagnetic energy, fast sampled between 23.2 and $24 \mathrm{~s}$.

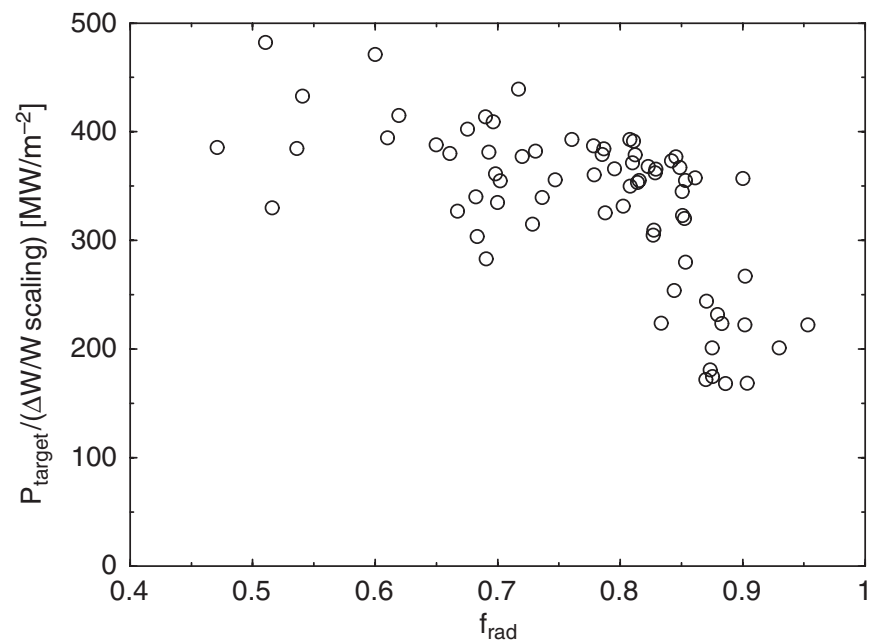

Figure 11. \#53318, ratio of power density to the outer divertor target to the predicted $\Delta W / W$ scaling, versus the radiative power fraction. 
in particular at high radiative power fractions $\left(f_{\text {rad }} \approx 90 \%\right)$. This seems to be an indirect indication of ELM mitigation. The fact that only at high radiative power fractions the ELM mitigation becomes effective is probably due to cooling rates characteristic of nitrogen. At high $f_{\text {rad }} \geqslant 0.85$, the electron temperature in front of the target is dropping below $15 \mathrm{eV}$ during the actual ELM event. At those electron temperatures of $10-15 \mathrm{eV}$, nitrogen radiates most efficiently (the cooling rate of nitrogen is increasing from 20 to $10 \mathrm{eV}$ by a factor of 10 [8]). This is consistent with calculations of ELM mitigation due to radiation [14], which predict for similar small ELMs $\left(\Delta W / A \approx 0.3 \mathrm{MJ} \mathrm{m}^{-2}\right)$ that the transient radiative loss can have the same magnitude as the conducted power leading to a reduction of the ELM power load by about a factor of 2. This of course would mean that ELM mitigation by nitrogen seeding is only possible with type-III ELMs, i.e. small ELMs with low energy densities $\triangle W / A$ as the above-mentioned ones, and that type-I ELMs cannot be mitigated by nitrogen seeding. For the mitigation of larger ELMs, seeding of elements with a higher $Z$ is needed. However, it remains to be proven if large amounts of a high- $Z$ element (such as argon) can be seeded without leading to unacceptable impurity concentrations in the plasma core.

In principle, under ELM mitigation one understands primarily the reduction of the heat flux from the pedestal to the target plates during the ELM event. Thus a proof of the ELM mitigation is only possible by a direct comparison of the energy lost at the pedestal $\Delta W_{\mathrm{ELM}} / W_{\text {ped }}$ and the power arriving at the $P_{\text {target }}$. The ELMy band (area between upper and lower envelope) in the power flux density measured by IR thermography at the divertor target is reduced, indicating ELM mitigation. However, reliable measurements of the energy loss during type-III ELMs are hardly feasible. The analysis of the fast diamagnetic energy signal does not resolve this problem. The ELM amplitude is lower than the noise level of the signal $(100 \mathrm{~kJ})$ and the inter ELM time is in a similar range as the characteristic time of the presence of eddy currents, which disturb the measurement. Another way of determining the energy loss by ELMs is measuring the kinetic energy loss in the plasma. By taking the electron temperature at the pedestal (ECE channel here at $R=3.685 \mathrm{~m}$ ) and the line-averaged density from an interferometer channel crossing the pedestal region, the kinetic electron energy can be deduced. This method is described in detail in [13]. The total energy loss per ELM derived is $\Delta W / W \approx 0.7 \%$ with an ELM frequency of $\approx 100 \mathrm{~Hz}(\# 53772, t=23.4 \mathrm{~s})$. Those values are in the range of other type-III ELM losses in discharges with impurity seeding [4,9]. However, the error bar in the determination of the kinetic energy loss is large due to the uncertainties in the assumptions about the relative contributions of electrons and ions as well as geometric uncertainties in the pedestal location. Hence, more detailed and more precise investigations in this respect are required in order to improve the understanding of mitigation techniques in the type-III ELM regime.

\section{Comparison of the Mk-II gas box divertor to the more open divertors with respect to nitrogen seeding}

In the previous sections, it was shown that an operation with a partially detached divertor is possible in the closed Mk-II gas box divertor. Energy and power balances are consistent, thus confirming the feasibility of type-III ELMy H-mode scenarios with about $90 \%$ radiative power fraction. The intention of the closed Mk-IIGB (installed in 1998) was to increase the divertor radiation with respect to the total radiation and to reduce the plasma core pollution with extrinsic impurities. For this reason, it is useful to compare the results obtained in the JET Mk-IIGB with the more open predecessors: the very open Mk-I (1994-1996), the more closed Mk-IIA and Mk-IIAP with plugged leakage paths (1996-1998). For the characterization of all divertors, an improved, consistent estimate of the total radiated power, based on tomographic 
reconstructions, is used. In order to minimize errors due to reconstruction artefacts in the divertor region and to increase reliability, not all divertor bolometer channels are used for the evaluation of the total radiated power within this algorithm. This algorithm was cross validated by an energy balance with thermocouple data in the Mk-IIGB divertor. A thorough re-evaluation of the total radiated power in nitrogen discharges has been made for all JET divertors, including the discharges in Matthews et al [4].

Figure 12 shows the maximum achieved radiated power fraction versus the ratio of divertor to bulk radiation for all divertors. Note that this ratio does not distinguish between radiation from divertor legs and radiation from the X-point region. First of all, increasing the radiative power fraction by seeding of nitrogen leads to an increased divertor radiation. This dependence is linear regardless of the divertor closure. Of course, this does not mean that the radiation in the divertor legs is increased. As shown in section 2, with increasing radiative power fraction the peak of the radiation distribution moves towards the X-point region, leading also to enhanced radiation inside the LCFS. This is similar to all other divertors [4]. The highest radiative power fraction was achieved in the Mk-I. Mk-IIA data are systematically lower than Mk-I and Mk-IIGB data. For Mk-IIAP, unfortunately only data from disrupted discharges were available. All the data from the disrupted discharges reveal the same linear dependence, but lead to an even larger scatter of the data. This scatter mainly reflects different machine operating areas, but does not reveal an operating boundary. This seems to suggest that the divertor closure has no effect on the maximum achievable radiative power fraction. Furthermore, there are strong indications that in several of the disruptive nitrogen seeded discharges the radiative power fraction just before the disruption is about $100 \%$. This suggests that the actual limit for those radiating scenarios is the radiative collapse, which is a global limit not depending on divertor geometry. More investigations at the $\mathrm{H}$ - to L-mode boundary are necessary to be able to give a clear answer to the operational limit of those radiation cooled discharges.

Previous conclusions [4], based on a power balance between bolometer data and Langmuir probe data, have to be modified somewhat. When comparing the maximum achieved radiative power fractions, derived by the new algorithm, for the Mk-I divertor (\#33204), with the results

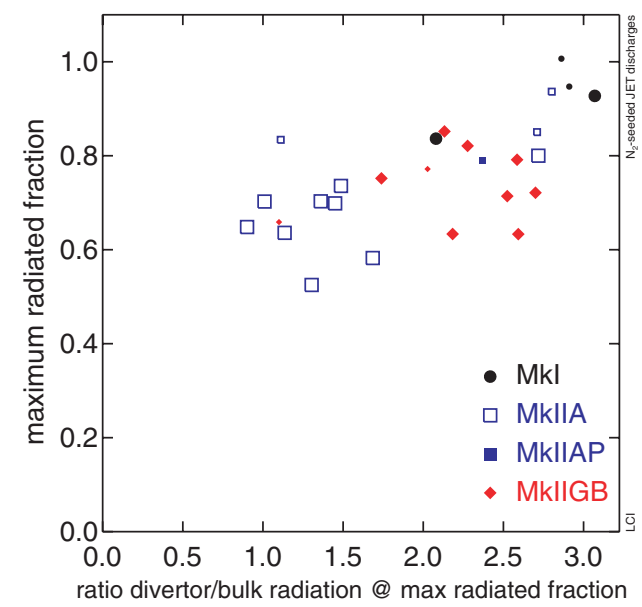

Figure 12. Comparison of the radiative power fractions achieved in JET divertors with nitrogen seeding as a function of the ratio of divertor to total radiation: Mk-I, Mk-IIA, Mk-IIAP, Mk-IIGB; small symbols indicate disrupted discharges, where the radiated powers were taken at arbitrary times before the actual disruption. 
Table 1. Effect of divertor closure on detachment process; DoD at inner divertor.

\begin{tabular}{lllc}
\hline Divertor & $f_{\text {rad }}\left(\mathrm{DoD}^{\text {int }} \geqslant 10\right)$ & $N^{\mathrm{GW}}\left(\mathrm{DoD}^{\text {int }} \geqslant 10\right)$ & DoD $^{\max }$ \\
\hline Mk-I & 80 & 70 & 10 \\
Mk-IIA & 65 & 65 & 20 \\
Mk-IIAP & 60 & 65 & 40 \\
Mk-IIGB & 55 & 65 & 100 \\
\hline
\end{tabular}

of the old analysis published in [4], it is obvious that less neutral particle loss needs to be assumed to achieve a consistent power balance. The previously assumed high level of neutral particle loss was not supported by EDGE2D/NIMBUS simulations in conjunction with more detailed bolometer analysis anyway [15]. So, it seems that the neutral particle loss is nonsignificant and can be neglected in the power balance for those highly radiating nitrogen seeded discharges.

As previously noted [4], the detachment becomes more pronounced as the divertor becomes more closed. The radiative power fraction at the onset of complete detachment $\left(\mathrm{DoD}^{\mathrm{int}} \geqslant 10\right)$ is decreasing with increasing divertor closure (see table 1). Similarly, the maximum achieved DoD is increasing significantly with increasing divertor closure. However, the onset of the detachment with respect to the Greenwald number $N^{\mathrm{GW}}=\bar{n}_{\mathrm{e}} / n_{\mathrm{e}}^{\mathrm{GW}}$ is unaffected by the divertor closure.

\section{Discussion}

In the previous sections, it has been shown that the steady state as well as the transient heat flux to the divertor target plates can be reduced significantly. However, the confinement of those discharges is reduced as well. For ITER, it has to be demonstrated that any exhaust technique is compatible with good confinement. The transition from type-I to type-III ELMs leads to a reduction in confinement down to $f_{\mathrm{H} 98} \approx 0.8$. The ITER operational domain $[16,17]$ does foresee an operation at lower confinement enhancement factors for higher densities. ITER has the capability for $Q=10$ operation at a density of $\bar{n}_{\mathrm{e}} / \bar{n}^{\mathrm{GW}} \approx 1.0$ and at a plasma current of $17 \mathrm{MA}\left(q_{95} \approx 2.65\right)$ with a confinement enhancement factor of $f_{\mathrm{H} 98} \approx 0.8$. Consequently, one has to demonstrate a successful operation at $\bar{n}_{\mathrm{e}} / \bar{n}^{\mathrm{GW}}=1, f_{\mathrm{H} 98}=0.8, q_{95}=2.6$.

There is potential to increase the operational range of the scenario presented in this paper to higher densities by increasing the triangularity. The discharges shown in this paper have triangularities of $\delta \approx 0.2$. It has been shown [18] that increasing triangularity leads also to improved confinement at high densities in type-III ELMy H-modes. Without impurity seeding, $f_{\mathrm{H} 98} \approx 0.8$ has been achieved with an average triangularity of $\delta=0.47$. Furthermore, there is potential to increase the stored energy by lowering the safety factor at the edge from 2.8 to 2.6. No principle problems (i.e. MHD) have been encountered at this low $q_{95}$ operation.

\section{Conclusions}

It has been shown that a radiation cooling scenario with radiative power fractions of more than $90 \%$ is possible at JET, regardless of the divertor closure. This type-III ELMy regime leads to a partially detached $\mathrm{H}$-mode at $85 \%$ of the Greenwald density and $\mathrm{H}$-factors of $f_{\mathrm{H} 98}=0.82$. The heat flux density is reduced to about $2-5 \mathrm{MW} \mathrm{m}^{-2}$ and electron temperatures in the divertor of less than $10 \mathrm{eV}$ are feasible. There is an indication of ELM mitigation at high radiative power fractions (90\%). However, more detailed investigations on the energy lost at the pedestal 
during type-III ELM activity are required in order to prove the effect of mitigation. Despite the uncertainties with respect to the ELM mitigation, this regime might offer a potential ITER scenario with a highly radiating plasma boundary and small ELMs.

\section{References}

[1] Greenwald M et al 1988 Nucl. Fusion 282199

[2] Samm U et al 1993 Plasma Phys. Control. Fusion 35 B167

[3] Kallenbach A et al 1995 Nucl. Fusion 351231

[4] Matthews G F et al 1999 Nucl. Fusion 3919

[5] Saibene G et al 2001 9th European Fusion Physics Workshop (Saariselkä, Finland, 10-12 December 2001)

[6] Neu R et al 1996 Plasma Phys. Control. Fusion 38 A165

[7] Philipps V et al 2000 Plasma Phys. Control. Fusion 421

[8] Post D E, Jensen R V, Tarter C B, Grasberger W H and Lokke W A 1977 At. Data Nucl. Data Tables 20397

[9] Strachan J D et al 2000 Plasma Phys. Control. Fusion 42 A81

[10] Eich T et al 2001 Proc. 28th European Physical Society Conf. on Controlled Fusion and Plasma Physics (Funchal, Portugal, 18-22 June 2001) Europhys. Conf. Abstr. vol 25a, ed C Silva, C Varandas and D Campbell (Geneva: European Physical Society) p 1809

[11] Herrmann A et al 1995 Plasma Phys. Control. Fusion 3717

[12] Loarte A et al 1998 Nucl. Fusion 38331

[13] Fishpool G M 1998 Nucl. Fusion 381373

[14] Loarte A et al 2000 IAEA-CN-77 ITERP/11(R) 18th IAEA Conf. (Sorrento, Italy, 4-10 October 2000) Fusion Energy 2000 (IAEA, Vienna, 2001) ISSN 1562-4153

[15] Ingesson L C 1997 Proc. 24th European Physical Society Conf. on Controlled Fusion and Plasma Physics (Berchtesgaden, Germany, 9-13 June 1997) Europhys. Conf. Abstr. vol 21a, part I, ed M Schittenhelm, R Bartiromo and F Wagner (Geneva: European Physical Society) p 113

[16] Aymar R 2001 Summary of the ITER Final Design Report

[17] Campbell D J 2001 Phys. Plasmas 82041

[18] Saibene G et al 2001 Proc. 28th European Physical Society Conf. on Controlled Fusion and Plasma Physics (Funchal, Portugal, 18-22 June 2001) Europhys. Conf. Abstr. vol 25a, ed C Silva, C Varandas and D Campbell (Geneva: European Physical Society) p 1749 